\title{
Prenatal evaluation of a fetus with trisomy 18 and additional balanced de novo $\operatorname{Rob}(13 ; 14)$
}

\author{
R. Leśniewicz ${ }^{1}$, R. Posmyk², I. Leśniewicz¹, S. Wołczyński ${ }^{1}$ \\ ${ }^{1}$ Department of Reproduction and Gynecological Endocrinology, Medical University of Białystok \\ ${ }^{2}$ Department of Clinical Genetics, Medical University of Białystok
}

\begin{abstract}
The main aim of this work is to present unusual case with full trisomy 18 and additional robertsonian translocation- Rob $(13 ; 14)$ detected through abnormalities found in prenatal ultrasound examination. A 26 years-old pregnant women with no family history of any reproductive failure underwent level II ultrasound screening in 19 weeks of gestation. Polyhydramnios, intrauterine growth retardation, hydrocephalus, enlarged lateral ventricles, club foot and cardiac defect were found. Amniocentesis was indicated considering the high likelihood of a chromosomal aberration. Abnormal karyotype was detected 46,XY,der(13;14)(q10;q10),+18. Karyotypes of parents were normal, what confirmed de novo origin of this aberration. Pregnancy was terminated. In postnatal examination fetus demonstrated intrauterine groth retardation and a lot of dysmorphic features characteristic for trisomy 18: microcephaly, prominent occiput, very low set and posteriorly rotated ears, hypertelorism, small mouth, small recessed mandible, a high narrow palate, broad nasal bridge, low-set ears, preauricilar skin appendage, clenched fingers clinodactyly of $\mathrm{V}^{\text {th }}$ fingers and club foot. In conclusion it is worth to say that our described fetus demonstrated rather typical for trisomy 18 ultrasonographic features. Balanced Rob $(13 ; 14)$ gives no phenotypic expression. Possible interchromosomal effect in complex chromosomal aberration formation such as Rob (13;14) with trisomy 18 was discussed.
\end{abstract}

Key words: trisomy 18, prenatal ultrasound findings, robertsonian translocation, interchromosomal effect.

\section{Introduction}

The frequency of various chromosomal rearrangements in the general human population varies from $1 / 625$ to $1 / 5,000$ [1]. Trisomy 18 , also known as Edwards syndrome (ES), is the second most frequent numerical aberrations in fetus. A prevalence is estimated for 1 in 8000 up to 1 in 3000 live births [2]. A full trisomy of chromosome 18 is responsible for majority $(95 \%)$ of ES. Familial translocations cause $4 \%$ and only $1 \%$ are mosaics. It is postulated that the incidence rate increases with advanced maternal age. Usually the extra chromosome is of maternal origin, with meiosis II errors occurring twice as frequently as meiosis I errors $[3,4]$.

Robertsonian translocations (Robs) are the most common chromosomal rearrangements in humans,

Correspondence: R. Leśniewicz, Dept. of Reproduction and Gynecological Endocrinology, Medical University of Bialystok, Marii Sklodowskiej-Curie Str. 24, 15-249 Białystok, Poland; tel.: (+4885) 7468818, e-mail: ryszard.lesniewicz@gmail.com occurring 1 per 1000 live births [5]. According to original definition, giving by Robertson in 1916, Rob results from the whole arm exchanges between two nonhomologous acrocentric chromosomes $(13 ; 14 ; 15$; $21 ; 22$ ) and produce a single metacentric chromosome [6]. 50\% of all Robs appear de novo [1]. Around 95\% of them arise during maternal meiosis $[7,8]$. Different combinations of possible Robs can be observed. Depending on their frequency of occurrence Robs are divided into common (Rob (13q14q) and Rob (14q21q)), and rare [1]. $\operatorname{Rob}(13 ; 14)$ is the most frequent finding and constitute $73 \%$ of all Robs [9]. A mutation rate for de novo $\operatorname{Rob}(13 ; 14)$ was calculated for $1.5 \times 10^{-4}$ [10].

Coincidence of Rob $(13 ; 14)$ with trisomy 18 was observed in offspring of Rob $(13 ; 14)$ carriers. Such rather uncommon finding was interpreted as complex interchromosomal effect, which hypothetically could take place during meiotic disjunction [11].

Obligatory second trimester prenatal ultrasound screening and rapid advances in available techniques have greatly improved the early detection of various congenital anomalies in fetus. 

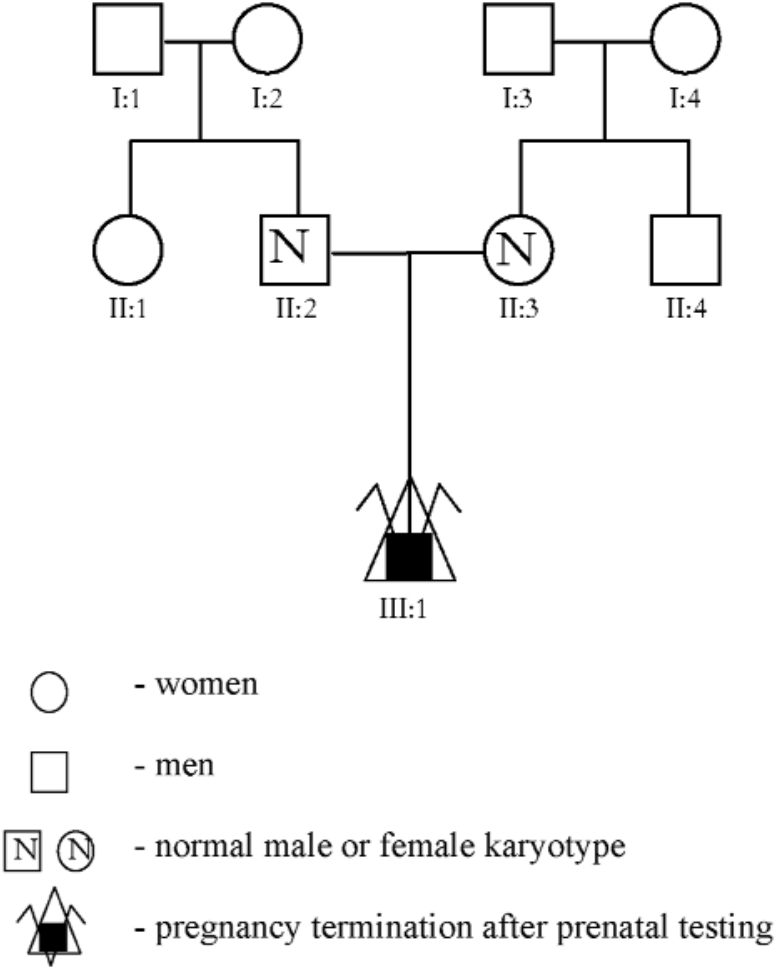

Fig. 1 Pedigree

\section{Case report}

A 26-year-old healthy woman, gravida 1, para 1, was refereed for prenatal cytogenetic diagnosis because of abnormal ultrasound findings. Family history for any reproductive failures was unremarkable (Fig. 1).

\section{Methods}

Ultrasound examination. The second trimester ultrasound examination was performed at 19 weeks' gestation at a specialty ultrasound unit. All essential biometrical parameters were measured.

Cytogenetics. Amniocytes were cultured using standard laboratory techniques. A total number of $30 \mathrm{GTG}$ banded metaphases were examined, with complete analysis and documentation preparation of four karyotypes (Fig. 2a-b).

\section{Results}

Ultrasound examination at 19 weeks' gestation demonstrated a singleton fetus with a fetal biometry equivalent to 16 weeks'. All measurements the BPD (biparietal diameter measured), HC (head circumference), AC (abdominal circumference), FL (femur length) were below suspected range, what confirmed IUGR (intrauterine growth retardation) in fetus. Additionally, polyhydramnios, hydrocephalus, enlarged lateral ventricles, club foot and cardiac defect were found (Fig. 3 a-c). The parents were informed of these suspicious sonographic findings and amniocentesis was indicated because of the high a
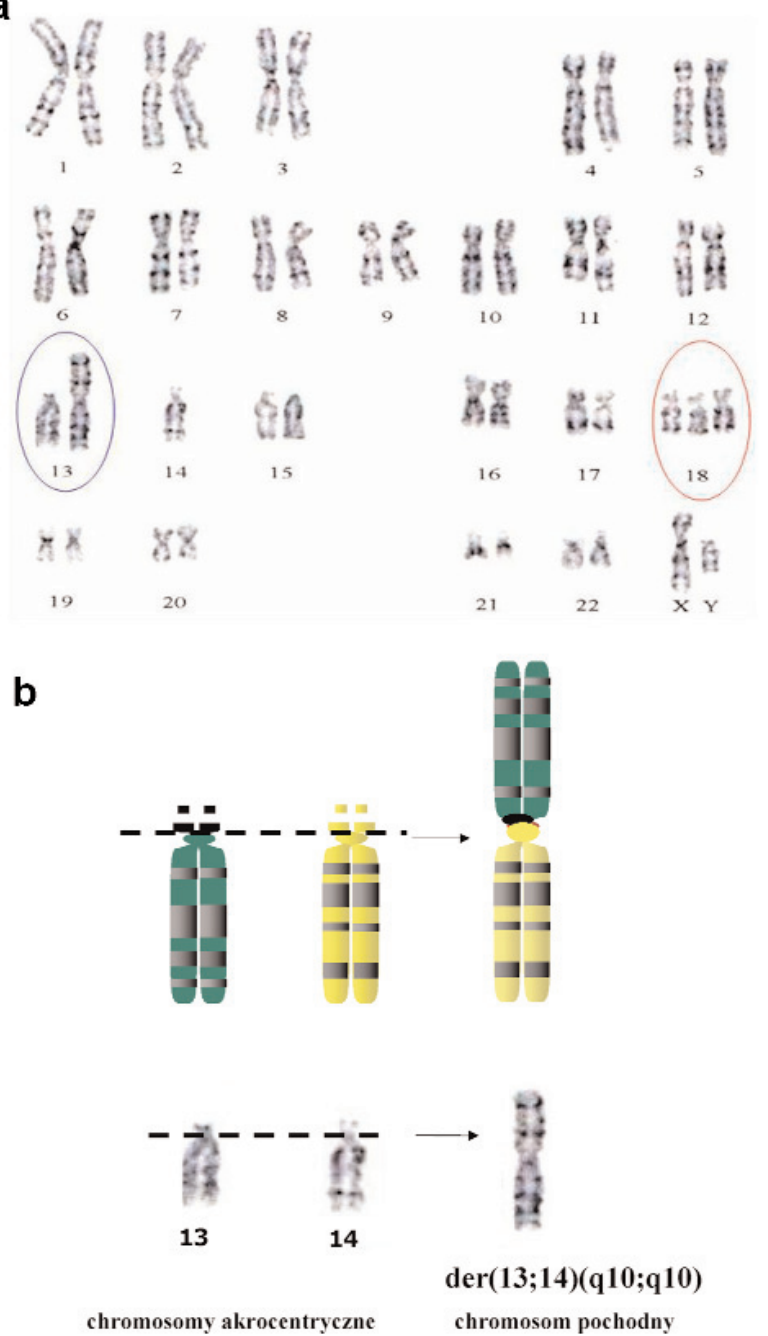

Fig. 2. a. Karyotype: $46, X Y, \operatorname{der}(13 ; 14)(q 10 ; q 10),+18$. b. Scheme of $\operatorname{der}(13 ; 14)(\mathrm{q} 10 ; 10 \mathrm{q})$ appearance

likelihood of a chromosomal aberration. Cytogenetic analysis of the cultured amniocytes revealed abnormal karyotype with balanced robertsonian translocation between nonhomologous acrocentric chromosomes 13 and 14 and additional trisomy 18 . Karyotype was described as: 46,XY,der(13;14) (q10;q10),+18 (Fig. 2a). Karyotypes of parents were normal, what confirmed de novo origin of this rather rare aberration. Edwards syndrome due to trisomy 18 was recognized in fetus. Genetic counseling was giving for the family. A detailed nondirective discussion with couple was very essential. Parents were provided with information according cytogenetic findings in fetus, diagnosis of Edwards syndrome. They were informed about pathophysiology, frequency, mortality, survival, prognosis according possible physical and mental status postnataly. Recurrent risk for further unbalanced offspring was estimated as very low. A couple elected to terminate the pregnancy. Labor was induced at 21 weeks of gestation. 

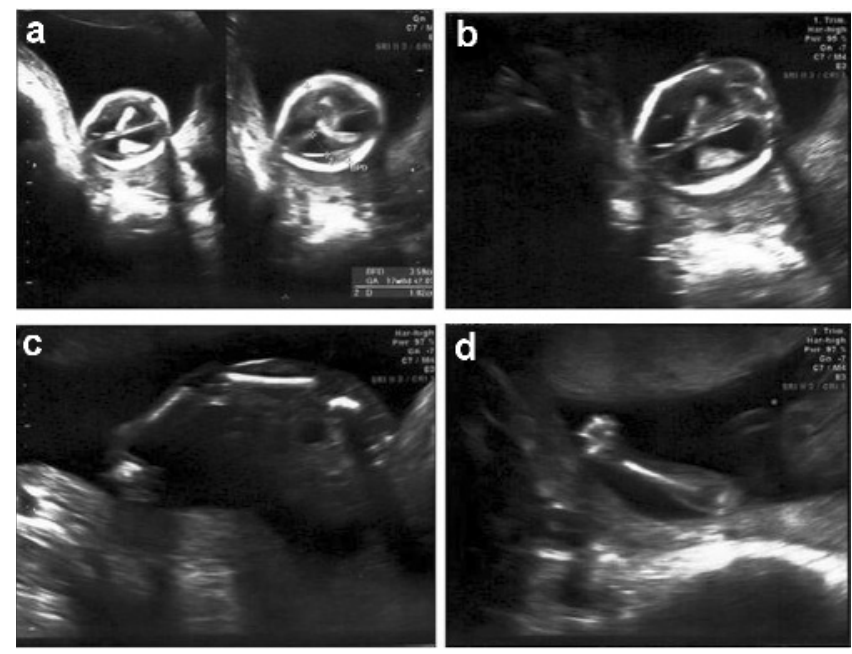

Fig. 3 Ultrasonographic findings: (a-b) hydrocephalus, enlarged lateral ventricles; (c-d) club foot.

Examination of the abortus revealed a male fetus which weighed $380 \mathrm{~g}(<3$ centile), measured $20 \mathrm{~cm}$ in crown-rump length and the head circumference measured 15.8 ( $<3$ centile). The proband also had: microcephaly, prominent occiput, hypertelorism, small mouth, small recessed mandible, high narrow palate, broad nasal bridge, low-set posteriorly rotated ears, preauricilar skin appendage, clenched fingers and clinodactyly of $\mathrm{V}^{\text {th }}$ fingers (Fig. 4 a-c).

\section{Discussion}

Since the second trimester ultrasonography became a screening examination for fetal aneuploidy, a greater sensitivity of unbalanced fetus detection is observed. The current case report presents the recognition of extremely rare complex chromosomal aberration consisting of rob $(13 ; 14)$ and additional trisomy 18 , detected through abnormal ultrasound findings. It is a huge value of an accurate examination, because unbalanced fetus was diagnosed in a young 26-yearsold, healthy woman, with unremarkable family history, at no risk for a nondisjunction event. According to series of publications, the sensitiveness in detection of aneuploidy was very variable. The highest score of $97 \%$ for trisomy 18 detection during second trimester screening was reached by De Vore [12]. Detection rate was highly depended of gestational age. The highest scores were achieved around 19 weeks' pregnancy [13].

The ultrasound findings in our observed fetus, particularly IUGR, polyhydramnios, hydrocephalus, enlarged lateral ventricles, clenched fingers, club foot and cardiac defect belong to characteristic manifestation of the trisomy 18 described by other authors $[14,15]$.
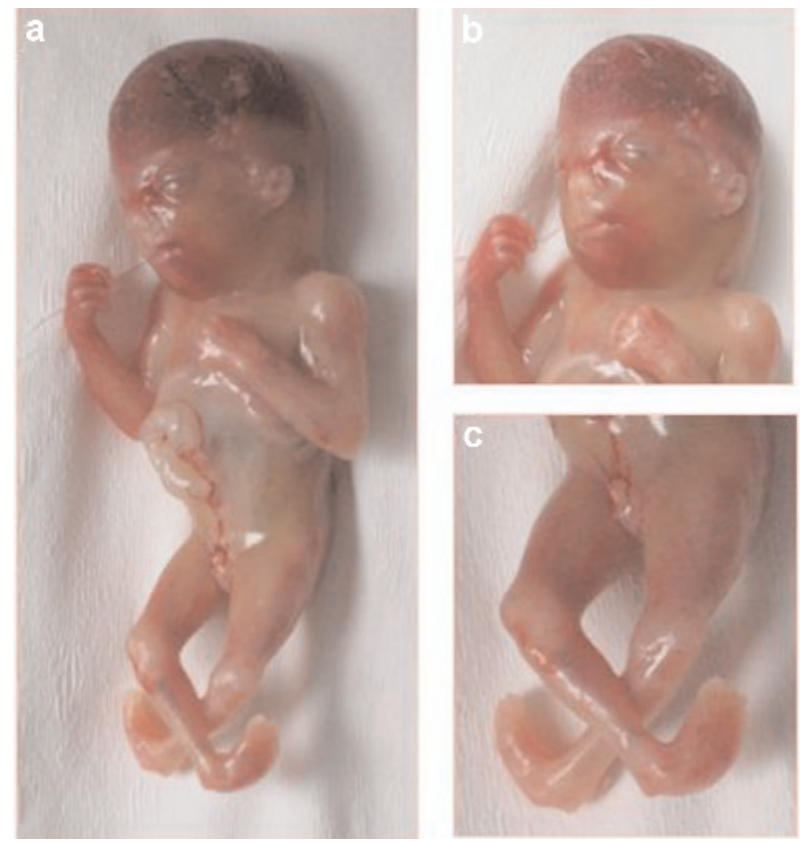

Fig. 4. Phenotype of abortus: microcephaly, prominent occiput, hypertelorism, small mouth, small recessed mandible, high narrow palate, broad nasal bridge, low-set posteriorly rotated ears, preauricilar skin appendage, clenched fingers and clinodactyly of $V^{\text {th }}$ fingers.

It is worth to stress a probable pathogenesis of rather uncommon karyotype in our found unbalanced fetus. Usually in trisomy 18 the extra chromosome is of maternal origin, with meiosis II errors occurring twice as frequently as meiosis I errors. It is also known that around $95 \%$ of Robs arises during maternal meiosis [7]. Several reports suggested that translocations disturb meiotic disjunction of chromosome pairs not involved in the translocation, resulting in additional trisomy offspring, what was named interchromosomal effect $[11,16,17]$.

In conclusion, the second trimester screening appears to have high accuracy for detection of trisomy 18 , especially around 19 weeks' pregnancy. Early prenatal detection of fatal chromosomal fetus is important for families, which may consider options regarding pregnancy continuation.

\section{References:}

[ 1] Shaffer LG, Jackson-Cook CK, Stasiowski BA, Spence JE, Brown JA. Parental origin determination in thirty de novo Robertsonian translocations. Am J Med Genet. 1992;43:957963.

[2] Jones KL. Smith's Recognizable Patterns of Human Malformation. $5^{\text {th }}$ ed. Philadelphia, PA: WB Sauders Co; 1997:14-17.

[3] Ya-gang X, Robinson WP, Spiegel R, Binkert F, Ruefenacht U, Schinzel AA. Parental origin of the supernumerary chromosome in trisomy 18. Clin Genet. 1993;44(2):57-61.

[ 4] Fisher JM, Harvey JF, Lindenbaum RH, Boyd PA, Jacobs PA. Molecular studies of trisomy 18. Am J Hum Genet. 1993;52(6):1139-44. 
[ 5] Hamerton JL, Canning N, Ray M, Smith J. A cytogenetic survey of 14,069 newborn infants: incidence of chromosomal abnormalities. Clin Genet. 1975;8:223-243.

[6] Robertson WMRB. Chromosome studies. J Morphol. 1916;27:179-331.

[ 7] Page SL, Shaffer LG. Nonhomologous Robertsonian translocations form predominantly during female meiosis. Nat Genet. 1997; 15:231-232.

[ 8] Bandyopadhyay R, Heller A, Knox-DuBois C, et al. Parental origin and timing of de novo Robertsonian translocation formation. Am J Hum Genet. 2002;71(6):1456-62.

[9] Therman E, Susman B, Denniston C. The nonrandom participation of human acrocentric chromosomes in Robertsonian translocations. Ann Hum Genet. 1989;53:49-65.

[10] Jacobs PA. Mutation rates of structural chromosome rearrangements in man. Am J Hum Genet. 1981;33:44-54.

[11] Munné S, Escudero T, Fischer J, Chen S, Hill J, Stelling JR, Estop A. Negligible interchromosomal effect in embryos of Robertsonian translocation carriers. Reprod Biomed Online. 2005; 10(3):363-9.
[12] DeVore GR. Second trimester ultrasonography may identify 77 to $97 \%$ of fetuses with trisomy 18 . J Ultrasound Med. 2000;19(8):565-76

[13] Bronsteen R, Lee W, Vettraino IM, Huang R, Comstock CH. Second-trimester sonography and trisomy 18. J Ultrasound Med. 2004;23(2):233-40

[14] Nicolaides KH, Snijders RJ, Gosden CM, Berry C, Campbell S. Ultrasonographically detectable markers of fetal chromosomal abnormalities. Lancet. 1992;340(8827):1109.

[15] Nyberg DA, Kramer D, Resta RG, et al. Prenatal sonographic findings of trisomy 18: review of 47 cases. $J$ Ultrasound Med. 1993;12(2):103-13.

[16] Lindenbaum RH, Hultén M, McDermott A, Seabright M. The prevalence of translocations in parents of children with regular trisomy 21: a possible interchromosomal effect? $\mathrm{J} \mathrm{Med}$ Genet. 1985;22(1):24-8

[17] Gianaroli L, Magli MC, Ferraretti AP, et al. Possible interchromosomal effect in embryos generated by gametes from translocation carriers. Hum Reprod. 2002;17(12):3201-7. 